\title{
High Spatial/Spectral Resolution Studies of Eta Carinae
}

\author{
Theodore R. Gull \\ Laboratory for Astronomy and Solar Physics, Code 681, Goddard Space \\ Flight Center, Greenbelt, MD 20771, USA
}

Eta Carinae HST Treasury Team

\begin{abstract}
We have used the high spatial and high spectral resolution of the Space Telescope Imaging Spectrograph (STIS) to study Eta Carinae and the Homunculus. Since the last minimum in 1998.0, CCD spectral modes have followed changes in the Eta Carinae, and large-scale changes in the Homunculus. Since 2001.7, MAMA echelle-mode observations have followed changes in the Eta Carinae and the very nearby ejecta through the 2003.5 minimum. Very significant changes in the star and nebular occur as the X-Ray drop occurs in the minimum.
\end{abstract}

\section{Introduction}

Eta Carinae is the best known Luminous Blue Variable (Davidson \& Humphreys 1999). The Homunculus (Gaviola 1950) is primarily a reflection nebulosity ejected during the outburst in the 1840's. It encloses the Little Homunculus (Ishibashi et al. 2003), an ionized shell, about one quarter the linear size of the Homunculus, consistent with ejecta from the 1890's, when a second brightening was noted. Weigelt B, C, and D (Weigelt \& Ebersberger 1986) are three bright emission knots within $0^{\prime \prime} 1$ to 0.3 northwest. The Central Source itself is highly variable both in brightness and line profiles. Damineli (1996) found a 5.52-year periodicity in the He I $10830 \AA$ line profile. The RXTE monitoring (Corcoran 2003) confirms the period to be $2023 \pm 7$ days. Modelling of CHANDRA spectroscopy (Pittard \& Corcoran 2002) shows that the hard x-ray radiation is due to wind-wind collisions between the $600 \mathrm{~km} \mathrm{~s}^{-1}$ wind and a $3000 \mathrm{~km} \mathrm{~s}^{-1}$ wind of a massive binary system. Ground-based observations of the star are heavily contaminated with velocity smeared nebular line emission. With the $0.05 /$ pixel sampling of the STIS CCD and spectral coverage from 1640 to $10200 \AA$, spectra of the Central Source were obtained apart from the Weigelt blobs. Zethson (2001) identified over 2000 emission lines from Weigelt blobs B and D, including many variable lines, especially Lyman- $\alpha$-pumped Fe II lines in the near ultraviolet, between the minimum of 1998.2 and the broad maximum in 1999.3 and 2000.2. Verner et al. $(2002,2003)$ used CLOUDY to model the Weigelt blob spectra including Fe II during the 1998 minimum and the maximum. Hillier et al. (2001) modelled the Central Source during the minimum. The IUE spectra recorded between 1977 and 1996 indicate spectral changes during the approximately one hundred day minimum. However, since the 5.52-year period was not known, the observations were intermittent in time. Moreover, the relatively 
large apertures of IUE $\left(3^{\prime \prime}\right.$ diameter and $10^{\prime \prime} \times 18^{\prime \prime}$ oval $)$ provided little information on the spatial structure other than half of the uv radiation passed through the smaller aperture.

With its high spatial and spectral resolutions, HST/STIS allowed us to separate the star from the nebulosity. The CCD spectroscopy and long apertures permitted sampling of the Homunculus to determine its three-dimensional shape and mapping of the several very different nebular structures, not possible from the ground. As we approached the minimum predicted to occur in 2003.5, a series of observations were successfully proposed to monitor the changes across the x-ray drop with RXTE, CHANDRA, HST/STIS, and the VLT/UVES. Much additional background information on Eta Carinae is in a conference proceedings (Gull, Johansson, \& Davidson 2001) and references cited therein.

\section{The STIS Observations}

Our systematic studies of Eta Carinae provided much new insight on the structure of the overall nebula, variations of the Central Source, and detailed structures in the immediate vicinity of the Central Source. The first MAMA echelle observations recorded of the Central Source demonstrated that the stellar emission is extended. Through the STIS $0.2 \times 0.2$ aperture, a nearly point source is present, with weak extensions. In addition to the interstellar lines, seen as absorption lines perpendicular to the spectral dispersion, other absorption lines are tilted across the spatial extent of the aperture, some with small velocity changes, and other broad lines are seen with large velocity changes. We chose to use a wider aperture, $0^{\prime \prime} 3 \times 0^{\prime \prime} 2$, to monitor the extended structure.

Between 2380 and $3160 \AA$, over 500 absorption lines with 20 velocity systems were identified. Most of the lines are from neutral and singly ionized iron-peak elements. Unlike ISM lines that originate from ground energy states, these lines originate from many higher energy states, some above $20,000 \mathrm{~cm}^{-1}$. Two velocity systems are of note: $-146 \mathrm{~km} \mathrm{~s}^{-1}$ and $-513 \mathrm{~km} \mathrm{~s}^{-1}$. The former appears to have energy populations consistent with $7000 \mathrm{~K}$ and the latter with $700 \mathrm{~K}$. During the minimum, a number of new velocity components appear between 170 and $-385 \mathrm{~km} \mathrm{~s}^{-1}$, and the strength of the $-146 \mathrm{~km} \mathrm{~s}^{-1}$ lines change. The -513 $\mathrm{km} \mathrm{s}^{-1}$ components do not change significantly. These systems are likely ejecta located in the wall of the foreground lobe of the Homunculus. The $-513 \mathrm{~km} \mathrm{~s}^{-1}$ component is at a considerable distance, likely 20,000 AU, from the Central Source. The $-146 \mathrm{~km} \mathrm{~s}^{-1}$ component is very close. The intermediate velocity components appear in Fe II lines during the minimum. During the minimum, the far and mid-uv radiation disappears, and these systems relax from Fe III to Fe II. Up to the x-ray drop, Fe III and Si III] emission can be seen, along with highly excited Fe II, close to the Central Source as depicted in Figure 1 recorded seven days before the x-ray drop on June 29 (Top). Seven days later (Bottom), both Fe III and Si III] emissions have virtually disappeared.

\section{Conclusions and Acknowledgements}

High spatial and high spectral resolution instruments are providing much new insight into the structure of Eta Carinae and its ejecta. The Eta Carinae ob- 

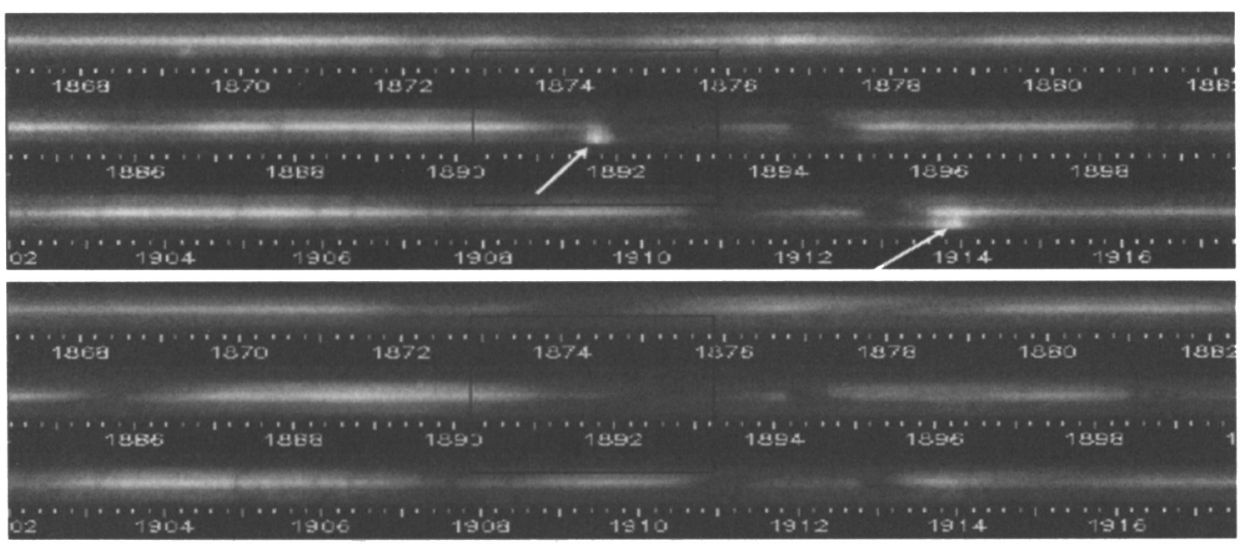

Figure 1. Top: June 22, 2003. Bottom: July 5 2003. Arrows point to Si III] and Fe III emission that disappears after x-ray drop

servations were accomplished through NASA and STScI under STIS GTO (T. Gull, P.I.) and HST GO (K. Davidson, P.I.) programs. We are indebted to Don Lindler and Keith Feggans for their systems and software support.

\section{References}

Corcoran, M. 2003, IAU Circ. 8160

Damineli, A. 1996, ApJ, 460, L49

Davidson, K., \& Humphreys, R. 1999, ARA\&A, 35, 1

Gaviola, E. 1950, ApJ, 111, 408

Gull, T., Johansson, S., \& Davidson, K. (editors) 2001, Eta Carinae \& Other Mysterious Stars, ASP, 242

Hillier, J., et al. 2001, ApJ, 553, 837

Ishibashi, K., et al. 2003, AJ, 125, 3222

Pittard, J. M., \& Corcoran, M. F. 2002, A\&A, 383, 636

Verner, E., et al. 2002, ApJ, 585, 115

Verner, E., et al. 2003, 592, 59

Weigelt, G., \& Ebersberger, J. 1986, A\&A, 163, L5

Zethson, T. 2001, Ph.D. Thesis, Univ of Lund 